\title{
Avaliação da frequência cardíaca e pressão arterial em mulheres praticantes de strong by zumba ${ }^{\mathrm{TM}}$
}

Heart rate evaluation and blood pressure in women who practise strong by zumba ${ }^{\text {mox }}$

Milena Araújo de Oliveira

Universidade Federal de Mato Grosso, Brasil

Anibal Monteiro de Magalhaes Neto

Universidade Federal de Mato Grosso, Brasil

Nayara Costa Araújo

Universidade Estadual de Campinas, Brasil

nayaranana_@hotmail.com

\section{Resumo:}

Strong by Zumba é a combinação de treinamento de alta intensidade com exercícios de força e movimentos base que visam um impacto metabólico e cardiorrespiratório. O objetivo deste estudo foi verificar a frequência cardíaca e a pressão arterial em mulheres durante a prática de Strong by Zumba ${ }^{\text {mix }}$. As atividades foram realizadas em uma feira coberta no interior do estado de Mato grosso, com 45 mulheres praticantes assíduas da modalidade com média de $41 \pm 5$ anos. Foram realizadas aferições de pressão arterial e frequência cardíaca antes, durante e após a atividade. Os resultados mostraram uma frequência cardíaca média inicial de 83,4 $\pm 17,9$ bpm, durante do exercício a média foi de 135,1 $\pm 18,8$ bpm e após a prática obteve-se a média de 118,3 $\pm 17,9$ bpm, caracterizando a aula como de intensidade vigorosa. A pressão arterial média inicial foi $88,4 \pm 8,4 \mathrm{mmHg}$, durante a seção de exercício a média foi de $88,6 \pm 10 \mathrm{mmHg}$ e a após o exercício $85,1 \pm 9,1 \mathrm{mmHg}$. Observa-se neste estudo que a prática regular de Strong by Zumba ${ }^{\mathrm{mx}}$ pode contribuir para a melhora da resposta cardiovascular e do condicionamento físico, se tornando uma modalidade favorável para a saúde de seus praticantes.

Palavras-chave: Dança, Condicionamento físico, Saúde.

\section{Abstract:}

Strong by Zumba ${ }^{n}$ is a combination of high intensity training with strength exercises and basic moves aimed at a metabolic and cardiorespiratory impact. The purpose of this study was to verify heart rate and blood pressure in women during Strong by Zumba ${ }^{\text {"w }}$ practice. The activities were held in a covered fair in the interior of the state of Mato Grosso, with 45 women aged $41 \pm 5$ who assiduously practise the dance. . Blood pressure and heart rate measurements were performed before, during and after the activity. The results showed a moderate initial heart rate of $83.4 \pm 17.9 \mathrm{bpm}$, an average of $135.1 \pm 18.8 \mathrm{bpm}$ during practice, and an average of $118.3 \pm 17.9 \mathrm{bpm}$ after practice, characterizing the class as of vigorous intensity. The initial arterial pressure was $88.4 \pm 8.4$ $\mathrm{mmHg}$, during the exercise section the average was $88.6 \pm 10 \mathrm{mmHg}$, and after exercise $85.1 \pm 9.1 \mathrm{mmHg}$. In this study, it was observed that the regular practice of Strong by Zumba ${ }^{m}$ can contribute to the improvement of cardiovascular response and fitness, becoming a favorable modality for the health of its practitioners.

KEYwORDS: Dance, Physical conditioning, Health.

\section{INTRODUÇÃO}

O exercício físico provoca uma série de respostas fisiológicas, resultantes de adaptações metabólicas e hemodinâmicas que influenciam todo o corpo. Estas alterações fisiológicas podem se dar por meio de mudanças na temperatura corporal, peso, frequência cardíaca, pressão arterial entre outras que podem ser utilizadas como parâmetros de avaliação de treinamento. Além disso, o exercício físico resulta em uma melhora da aptidão cardiorrespiratória, força muscular, composição corporal, resistência muscular e flexibilidade melhorando o condicionamento físico do praticante (Triani, Lima, Neto \& Monteiro, 2018).

\section{Recepción: 09 de agosto de 2019 | Aprobación: 21 de mayo de 2020 | Publicación: 1 de junio de 2020}


Dentre os vários tipos de exercícios físicos existentes, destaca-se a dança, que assim como qualquer outra atividade física, promove um aumento do dispêndio energético melhorando a aptidão física, saúde cardiovascular, coordenação e equilíbrio, bem como força, flexibilidade, capacidade aeróbica e propriocepção (Araújo et al., 2017). Além disto, a dança como atividade em grupo pode ajudar a melhorar a autoestima e as relações sociais e afetivas, contribuindo no processo de desenvolvimento interpessoal e comunicativo (Barretoi, 2011; Oliveira et al., 2017).

Nos últimos anos a dança tem se destacado em academias de ginásticas e nos programas de atividades físicas, surgindo diversas modalidades como a $\mathrm{Zumba}^{1}{ }^{1}$ que associa os princípios básicos do treinamento físico aeróbico e fortalecimento, por meio de passos coreografados pelo professor (Ljubojević, Jakovljević \& Popržen, 2014). Em 2016 o grupo idealizador da Zumba criou o Strong by Zumba ${ }^{\mathrm{Tx}}$, que incorpora o método de treino intervalado de alta intensidade conhecido como HIIT e exercícios combinados de força e movimentos base, que visam um impacto metabólico e cardiorrespiratório alto, utilizando resistência progressiva, sobrecarga, pliometria, treino funcional focado no core, ou na utilização do peso corporal, recorrendo a ritmos fortes e animados (Vlatch, 2017; Strong, 2017). No entanto, este novo programa de atividade física não se encaixa dentro da modalidade de dança, e sim de treinamento físico aeróbico, uma das características que o diferencia da Zumba ${ }^{\circ}$.

Em suma, o Strong by Zumba ${ }^{\text {mu }}$ é um programa de intensidade intermitente ou intervalada, no qual movimentos de alta intensidade (tais como elevação dos joelhos, polichinelos, etc.) são intervalados com movimentos de baixa intensidade (como lunges (avanços), pular corda, kickboxing) durante toda a aula (Strong, 2017). Sua música foi criada especificamente para o programa e passou por uma engenharia reversa para combinar com os movimentos e proporcionar uma sincronia perfeita durante uma aula com duração entre 55 e 60 minutos composta por um aquecimento, quatro segmentos principais da aula chamados de Quadrantes, cada qual com um objetivo específico, escala de intensidade, seleção de exercícios e um relaxamento. Após cada Quadrante há uma sessão de Recarregar de 30 a 90 segundos, para se fazer a transição entre Quadrantes.

Vários estudos têm demostrado que a prática do Zumba reduz significativa os níveis de pressão arterial e triglicerídeos em seus praticantes (Araneta \& Tanori, 2015; Cugusi et al., 2016) e melhora o desenvolvimento aeróbio e aptidão cardiovascular (Vendramin et al., 2016). No entanto, apesar de vários estudos apontando os efeitos da Zumba no condicionamento físico de seus praticantes, estudos referentes as respostas cardiovasculares do Strong By Zumba ${ }^{\text {twx }}$ são escassos (Valente, 2018), fazendo-se necessários novos estudos a fim de elucidar seus efeitos no organismo e preencher a lacuna cientifica existente.

Em busca dessa compreensão, a questão que norteou essa pesquisa foi: Qual o comportamento da frequência cardíaca e da pressão arterial em mulheres praticantes de Strong By Zumba ${ }^{\mathrm{m} x}$ ? Assim, o objetivo deste estudo foi verificar as respostas cardiovasculares da Frequência Cardíaca (FC) e da Pressão Arterial (PA) em mulheres voluntárias praticantes da modalidade de Strong By Zumba ${ }^{\text {nut }}$, bem como analisar a relação entre o nível de atividade física e a FC e PA das participantes da pesquisa afim de verificar seu condicionamento físico.

\section{Metodologia}

\section{Tipo de pesquisa}

Trata-se de uma pesquisa quantitativa do tipo descritiva que segundo Triviños (1987), é uma pesquisa que exige do investigador uma série de informações sobre o que deseja pesquisar. Esse tipo de estudo pretende descrever os fatos e fenômenos de determinada realidade. $\mathrm{O}$ foco essencial destes estudos reside no desejo de conhecer a comunidade e seus traços característicos. 


\section{Amostra}

Foram avaliadas 45 mulheres com $46 \pm 11,5$ anos de idade, participantes assíduas da atividade realizada na Feira do produtor Olívio Scapini, onde praticavam aulas da modalidade Strong By Zumba ${ }^{\mathrm{ma}}$ duas vezes na semana que eram realizadas nas terças e quintas-feiras das 13:00 às 14:00 horas, e das 18:00 às 19:00 horas respectivamente, na quadra coberta da Feira. $\mathrm{O}$ total de participantes inscritas no projeto eram de 48 alunas, mas alguns participantes no momento da coleta dos dados não estavam presentes ou foram excluídas do estudo por não atender aos critérios de inclusão que foram: ter idade maior que 18 anos, ter disponibilidade para a participação no programa, praticar a modalidade a pelo menos um ano, não ser hipertensa ou tomar medicamentos que interfiram no controle da pressão arterial, responder aos questionários até o fim, participar de forma ativa da atividade proposta não se ausentando da aula durante a avaliação da frequência cardíaca, aceitar participar voluntariamente do estudo e assinar o Termo de Consentimento Livre e Esclarecido (TCLE). O projeto foi apresentado e aprovado pelo comitê de ética de pesquisa com seres humanos com o registro $\mathrm{n}^{\circ} 2.203 .073$.

\section{Caracterização da aula}

As aulas foram ministradas por uma instrutora credenciado e com experiência de 3 anos na condução desta modalidade. A duração completa da aula foi de 57 minutos e 64 segundos. As aulas foram compostas por um aquecimento, quatro segmentos principais da aula chamados de quadrantes, cada qual com um objetivo específico, escala de intensidade, seleção de exercícios e um relaxamento. No quadrante 1 utilizouse exercícios afim de aumentar a frequência cardíaca para manter uma intensidade sólida e moderada durante toda a atividade. No quadrante 2 utilizou-se de 1 a 2 intervalos HIIT por música e movimentos de recuperação ativa entre as Sequências. No quadrante 3 incluiu-se dois intervalos HIIT por música com movimentos de recuperação ativa depois de cada intervalo e no quadrante 4 utilizou-se somente exercícios executados no chão conforme protocolo de aula estabelecido no manual de instrutor do Strong by Zumba ${ }^{\text {Tu}}$. Após cada quadrante houve uma sessão de Recarregar de 30 a 90 segundos, entre a transição de um quadrante ao outro (quadro 1). Para este estudo não fez parte da contagem de tempo as faixas de ativação funcional e a de retorno calma, o tempo de aula utilizado como controle foi de 36 minutos e 2 segundos despendido por diversos quadrantes. As faixas e quadrantes descritos no quadro abaixo indicam os movimentos que foram utilizados em cada quadrante/coreografia, sendo que entre os exercícios podem existir diferentes combinações ao longo dessa mesma coreografia/quadrante (quadro 2). Assim sendo, apesar de cada parte musical ter o seu(s) passo(s), estes podem ir alterando a dificuldade/sequência a cada próxima vez que se repete a mesma parte musical. 
QUADRO 1

Configuração da aula de Strong by Zumba ${ }^{\mathrm{mx}}$

\begin{tabular}{|l|c|c|}
\hline \multicolumn{1}{|c}{ Nome do segmento } & Segmento & Tempo \\
\hline Aquecimento & Quadrante 1: & 8 a 12 min \\
\hline Acionar & Transição 1 & 30 a 90 seg \\
\hline Recarregar & Quadrante 2 & 8 a 12 min \\
\hline Intensificar & Transição 2 & 30 a 90 seg \\
\hline Recarregar & Quadrante 3 & 8 a 12 min \\
\hline Desafiar seus limites & Transição 3 & 30 a 90 seg \\
\hline Recarregar & Quadrante 4 & 8 a 12 min \\
\hline No chão & Transição 4 & 30 a 90 seg \\
\hline Recarregar & Relaxamento & 4 a 6 min \\
\hline Relaxamento &
\end{tabular}

Fonte: Manual Strong by Zumba ${ }^{\text {mix }}$ (2017)

\section{QUADRO 2}

Descrição das atividades que foram utilizadas em cada quadrante durante a aula de Strong by Zumba ${ }^{\mathrm{rx}}$

\begin{tabular}{|c|c|}
\hline Quadrante & Movimentos utilizados \\
\hline Ignição & $\begin{array}{l}\text { Joelho ao peito, saltos equiparados a saltos à corda, avanços unilaterais do pé, } \\
\text { agachamentos, rotaçōes do tronco com M.I. em isometria, pranchas, flexões, } \\
\text { escaladores, passos em "v" com meio agachamento, polichinelos, joelho ao cotovelo } \\
\text { contrário, socos de cotovelo, deslocamentos laterais, socos laterais, saltos com } \\
\text { deslocamentos laterais, uppercuts, afundos/lunges, chutos laterais, press de ombros, } \\
\text { abduções dos M.I., socos á frente com rotação do tronco, socos cruzados em direção } \\
\text { ao joelho do lado contrário e flexões na vertente de press de ombros. }\end{array}$ \\
\hline Recperação 1 & Step touch com variações, deslocamentos ao lado, frente e atrás. \\
\hline Combustão & $\begin{array}{l}\text { Step touch com rotação do tronco, saltos de skatters, agachamentos, passo de } \\
\text { capoeira, burpees, deslocamentos laterais, joelho ao peito, agachamento com salto, } \\
\text { uppercuts, corrida no lugar, afundos/lunges cruzados atrás, agachamento sumo, } \\
\text { rotações do tronco, pranchas, escaladores, mountain climbers, e deslocamentos à } \\
\text { frente e atrás. }\end{array}$ \\
\hline Recuperação 2 & Step touch com variações, deslocamentos ao lado, frente e atrás. \\
\hline $\begin{array}{c}\text { Desafie seus } \\
\text { limites }\end{array}$ & $\begin{array}{l}\text { Step touch, saltos a pés juntos à frente, skipping baixo para trás, pranchas, joelho ao } \\
\text { peito cruzado, rotação do tronco equivalente a uma "tacada" de basebol, } \\
\text { afundos/lunges laterais, deslocamentos laterais, socos laterais, corrida no lugar, } \\
\text { skipping alto, saltos de skatters, agachamentos, saltos com elevação do joelho, } \\
\text { elevação do calcanhar atrás, deslocamentos à frente e atrás, socos em direção ao chão, } \\
\text { afundos/lunges com salto, agachamentos sumo com elevaçōes dos calcanhares, } \\
\text { skipping baixo no lugar e afundos/lunges atrás. }\end{array}$ \\
\hline Recuperação 3 & Step touch com variaçōes, deslocamentos ao lado, frente e atrás. \\
\hline Jogo de chão & $\begin{array}{l}\text { Em quatro apoios (cotovelos e joelhos no chão) um M.I. de cada vez, contração do } \\
\text { glúteo e extensão da coxa com o membro a } 90 \text { graus. Ainda na mesma posição, } \\
\text { adução e abdução do M.I. em extensão. Em quatro apoios (mãos e cotovelos) prancha } \\
\text { com rotação interna e externa dos M.S. fletidos e pranchas laterais alternadas. Em } \\
\text { decúbito ventral, contração do glúteo com extensão simultânea das coxas. }\end{array}$ \\
\hline
\end{tabular}




\section{Coleta de dados}

Para a coleta da frequência cardíaca, foi utilizado o relógio monitor cardíaco da marca polar, modelo FS1 BLU, registrado pelo número 80093269001 pela Anvisa. Para as aferições da pressão arterial, foi utilizado o aparelho de pressão digital de pulso da marca Premium, modelo LP200, registrado pela Anvisa pelo número 80275310048 .

Antes de começar qualquer atividade, foi pedido para que o participante fizesse a leitura do termo de consentimento livre esclarecido, se caso concordasse em colaborar com a pesquisa, era realizado a assinatura do TCLE e só depois iniciou-se a pesquisa. Utilizou-se para este estudo apenas uma aula de Strong by Zumba ${ }^{\mathrm{Tx}}$. $\mathrm{E}$ as coletas foram realizadas 30 minutos antes de iniciar a atividade, 30 minutos após o início e 30 minutos e após o termino da aula. Para a coleta dos dados foram colocadas nas participantes a cinta do monitor cardíaco. Em seguida, foi solicitado as participantes que ficassem sentadas para aferição da Frequência Cardíaca (FC) e Pressão Arterial (PA) iniciais.

Depois da coleta inicial, as participantes realizaram as atividades normalmente e depois de 30 minutos, foi efetuada uma nova coleta da FC e da PA e 30 minutos depois ao final da aula de Strong By Zumba ${ }^{\text {rx }}$ foram realizadas as coletas finais da FC e da PA. Após esses três momentos de coleta, a cinta do frequencímetro cardíaco foram retiradas pela pesquisadora.

No final de todo o processo de aferições, foi respondido o questionário internacional de atividade física versão curta. Foi escolhido o questionário IPAQ versão curta pelo fato de ser um questionário validado internacionalmente, de fácil aplicabilidade e entendimento, e que não demanda de muito tempo para dar respostas as perguntas, pois se trata de um questionário relativamente pequeno. Este questionário classifica os participantes em cinco categorias conforme a condição de atividade física: sedentário, insuficientemente ativo $\mathrm{A}$, insuficientemente ativo $\mathrm{B}$, ativo e muito ativo. Foi solicitado ainda que as participantes classificassem a sua saúde como regular, boa ou excelente.

\section{Análise estatística}

Para análise do questionário utilizou-se a estatística descritiva de frequência, utilizando as medidas de tendência central (média), medidas de dispersão (desvio-padrão) e frequência relativa. A distribuição de probabilidade dos dados analisados foi avaliada a partir do teste de normalidade Shapiro-Wilk. Os valores das variáveis $\mathrm{FC}$ e PA foram expressos em média e desvio padrão. As medidas das variáveis foram comparadas utilizando-se a análise de variância ANOVA para medidas repetidas e o grau de significância adotado foi de $\mathrm{p}<0,05$.

\section{Resultados e discussão}

No presente estudo explorou-se a resposta cardiovascular a uma aula de Strong By Zumba ${ }^{\mathrm{Tx}}$, em condições não laboratoriais por meio da análise da frequência cardíaca e pressão arterial. Participaram da pesquisa 45 mulheres com média de $46 \pm 11,5$ anos de idade, peso 72,24 $\pm 12,7 \mathrm{~kg}$, e estatura de 1,65 $\pm 0,3 \mathrm{~m}$. Dessas 45 participantes, 25 trabalham (55.5\%) e 20 não tinham emprego de forma remunerada (44,5\%). A média da jornada de trabalho das mulheres empregadas era de 7,1 horas por dia. Quanto ao nível de escolaridade, 25 não terminaram o ensino fundamental (55,5\%), 7 concluíram o ensino fundamental (15,6\%), 6 possuem ensino médio incompleto (13,3\%), 6 já concluíram o ensino médio $(13,3 \%)$ e apenas uma possui pósgraduação $(2,2 \%)$. Foi pedido para as participantes que avaliassem de forma geral como elas classificam a sua saúde, 
regular, boa ou excelente. 36 participantes responderam como regular ( $80 \%), 2$ como boa $(4,4 \%)$ e 7 pessoas classificaram como excelente (15,6\%).

No que diz respeito aos dados da FC, a média obtida antes da prática do exercício físico foi de 83,4 $\pm 17,9$ bpm, durante do exercício foi encontrado a média de 135,1 \pm 18,8 bpm e após a prática obteve-se a média de $118,3 \pm 17,9 \mathrm{bpm}$. Os dados apresentaram diferença significativa $(\mathrm{P}=0,001)$ durante e após o exercício físico quando comparado com a primeira avaliação (Figura 1).

\section{FIGURA 1}

Frequência cardíaca média e desvio padrão das praticantes de Strong By Zumba ${ }^{\mathrm{Tx}}$ antes, durante e depois do exercício físico. * Diferença significativa de $\mathrm{P}<0,05$

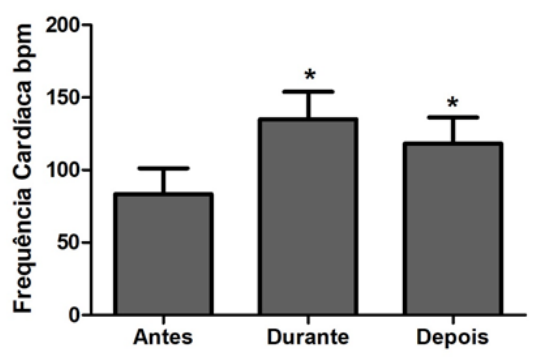

Fonte: Elaboração própria

Em relação a análise da Pressão Arterial Média (PAM), foi utilizada a seguinte equação: PAM= PAS + $(\mathrm{PAD} \times 2) / 3$. Os resultados mostraram uma PAM inicial de $88,4 \pm 8,4 \mathrm{mmHg}$, durante a seção de exercício encontrou-se a média de $88,6 \pm 10 \mathrm{mmHg}$ e a PAM final encontrada foi de $85,1 \pm 9,1 \mathrm{mmHg}$. Os dados não apresentaram diferença significativa entre os grupos durante e após o exercício quando comparado com a primeira avaliação, conforme ilustrado na Figura 2.

FIGURA 2

Pressão Arterial Média das praticantes de Strong By

Zumba $^{\text {nu }}$ antes, durante e depois da seção de exercícios.

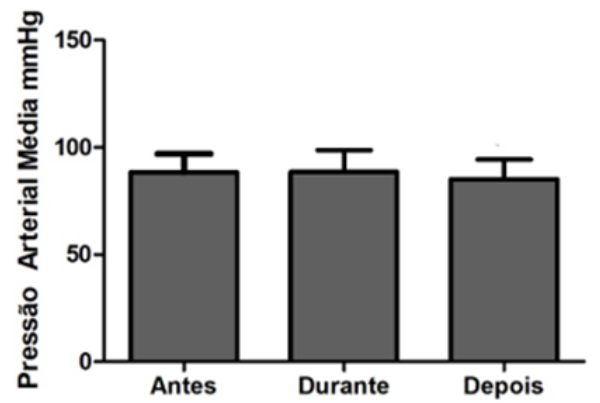

Fonte: Elaboração própria.

A primeira pergunta do questionário diz respeito a quantidade de dias da semana que o entrevistado pratica atividade física vigorosa, a média encontrada para essa pergunta foi de 3,2, em relação a duração destas atividades, a média obtida foi 1 hora. Quando perguntado a quantidade de dias da semana que a entrevistada pratica atividade física moderada, a média foi de 4,3, com uma média de duração de 5 horas. Foi perguntado quantos dias da semana elas fazem caminhada de no mínimo 10 minutos, a média obtida foi de 3,3, em relação ao tempo que elas gastam caminhando a média encontrada foi de 1,5 horas por semana. Foi questionado ainda quanto tempo elas gastam sentadas durante o meio de semana, obteve-se a média de 3,4 horas, já quando questionado o tempo que elas gastam sentadas durante o final de semana foi encontrado uma média de 4,5 horas (Tabela 1). De acordo com a classificação do nível de atividade física do IPAQ, a média obtida neste estudo classifica a população como ativa. 
TABELA 1

Média e desvio padrão das repostas do questionário IPAQ aplicado as praticantes de Strong By Zumba ${ }^{\text {Tx }}$

\begin{tabular}{clcc}
\hline Questão & \multicolumn{1}{c}{ Pergunta } & Média & $\begin{array}{c}\text { Desvio } \\
\text { Padrão }\end{array}$ \\
\hline 1A & Quantos dias da semana pratica atividade física vigorosa? & 3,3 & 1,1 \\
1B & Quanto tempo duram essas atividades em cada seção? & 1 & 0 \\
2A & Quantos dias da semana pratica atividade fisica moderada & 4,3 & 0,7 \\
2B & Quanto tempo duram essas atividades? & 5 & 0 \\
3A & Quantos dias da semana caminha pelo menos 10 min? & 3,3 & 1,1 \\
3B & Quanto tempo gasta caminhando? & 1,5 & 0,6 \\
4A & Quanto tempo gasta sentada durante o meio de semana? & 3,4 & 1,2 \\
4B & Quanto tempo gasta sentada durante o final de semana? & 4,5 & 0,8 \\
\hline
\end{tabular}

Fonte: Elaboração própria

Vale ressaltar que em algumas perguntas, as voluntárias não pontuaram, por exemplo na questão $2 \mathrm{a}, 36$ pessoas (80\%) não realizam atividades moderadas durante nenhum dia da semana. Na questão 3a, 31 pessoas (69\%) não caminham nenhum dia da semana. Na pergunta 4a, 2 voluntárias (4,4\%) não gastam nenhuma hora sentada durante a semana e por último na questão $4 \mathrm{~b}, 1$ voluntária $(2,2 \%)$ não gasta nenhuma hora sentado durante o final de semana. As pessoas que não pontuaram nessas questões não foram incluídas na média apresentada na Tabela 1.

Avaliou-se a resposta cardiovascular utilizando a FC por ser um modelo de avaliação eficiente como indicador de intensidade do exercício aeróbico e da aptidão cardiovascular, devido a relação com a capacidade aeróbica. Além da sua monitoração ser fácil e prático, visto que não é um método evasivo (Kraemer, Fleck \& Deschenes, 2015).

Com base nos resultados da FC média encontrados neste estudo, foi possível verificar a intensidade da aula Strong By Zumba ${ }^{\text {rxx }}$. Os dados apontaram para uma FC média mais alta de 135,6 bmp, tendo em vista que a média de idade encontrada nas participantes foi de 46 anos, ao utilizar a equação 220 - idade tem-se o valor da FC máxima (Heil, 2001). De acordo com o cálculo, o resultado da FC média corresponde a 78\% da FC máxima, que segundo a American College of Sports Medicine (ACSM) é classificada como intensidade vigorosa (ACSM, 2011; Garber et al., 2011).

Estudos avaliando a FC de praticantes de Strong By Zumba ${ }^{\text {atw }}$ são escassos. Até onde sabemos há apenas um estudo que comparou a FC de praticantes de Strong By Zumba ${ }^{\text {ra }}$ e praticantes assídua de zumba fitness, que encontraram uma FC média de 149,46 \pm 12,12 bpm (Valente, 2018), valor próximo do encontrado neste estudo.

Os resultados mostram que a PAM das participantes está de acordo com os valores indicados como normais pela Sociedade Brasileira de Cardiologia (SBC), sendo considerado normotensos pessoas cuja PAS $(\mathrm{mmHg}) \leq 120$ e PAD $(\mathrm{mmHg}) \leq 80$, ou PAM de $\leq 93,3$, (Malaquias, 2016), tornando assim a média do grupo estudado como normotensos.

Um estudo feito por Roy e Mandal (2017) buscou avaliar o efeito da intervenção de quatro semanas de Strong by Zumba ${ }^{\text {tx }}$ na circunferência abdominal e relação cintura e quadril em mulheres sedentárias e revelou que houve uma diminuição significativa na relação cintura-quadril das participantes avaliadas após a intervenção, refletindo em uma diminuição da gordura abdominal destas mulheres. Sabe-se que a obesidade representa um risco aumentado de desenvolver doenças cardiovasculares, hipertensão, colesterol entre outras (Yatsuya et al. 2014; Dada et al., 2018). Correlacionando esses dados com os achados deste estudo, podese pressupor que a prática do Strong By Zumba ${ }^{\text {Tx }}$ pode ser uma boa ferramenta no controle do sobrepeso e consequentemente da reposta cardiovascular e pressão arterial, porém isto precisa ser verificado.

Não foram encontrados na literatura estudos que analisaram a pressão arterial de praticantes de Strong By Zumba ${ }^{\text {rx }}$, diante disso, comparou-se os resultados desta pesquisa com a modalidade de Zumba. fitness. e Tanori (2015) em seu estudo com mulheres obesas com quadros de síndrome metabólica concluíram que o programa de treino de Zumba. promoveu a redução da pressão arterial e dos níveis de triglicerídeos nas praticantes. Apesar deste estudo não ter acompanhado a médio prazo a cinética dos níveis pressóricos durante várias sessões de treinos da modalidade, e de não ter apresentado diferença significativa, os resultados indicam uma diminuição de \pm 3,1 mmHg na Pressão Arterial Média (PAM) após a intervenção (Figura 2), indicando uma redução dos níveis pressóricos pós exercícios. 
Estudos de Pontes Junior, Prestes, Leite e Rodriguez (2011) sobre o exercício aeróbico, verificaram o efeito positivo desse tipo de exercício sobre a hipertensão e seus mecanismos fisiopatológicos. Os autores apontam que dentre os processos envolvidos no efeito anti-hipertensivo relacionados a essa modalidade de exercício, tem-se a diminuição da atividade simpática, aumento da sensibilidade barorreflexa e a melhora na função endotelial. Tendo em vista que o treino aeróbico é um dos principais componentes do Strong By Zumba ${ }^{\mathrm{rax}}$, é possível pressupor que a prática contínua desta modalidade a longo prazo pode contribuir para a manutenção da pressão arterial de seus praticantes. No entanto, vale destacar que até onde sabemos, este é o primeiro estudo abordando a resposta cardiorrespiratórios de uma aula de Strong By Zumba ${ }^{\mathrm{Tw}}$, frente a isso, novo estudos devem ser realizados para confirmar essa hipótese.

Importante destacar ainda que a maioria das participantes do estudo eram praticantes assíduas da modalidade de Strong By Zumba ${ }^{\text {ma }}$ e boa parte tinham uma vida ativa, conforme evidenciado no gráfico 3 referente ao nível de atividade física e qualidade de vida das participantes, de acordo com os resultados do questionário IPAQ versão curta aplicado no final da prática da atividade, o que justifica a adaptação ao treino encontrada neste estudo. Segundo Kokkinos e Narayan (2019), indivíduos com aptidão física moderada ou alta tem valores de PAS / PAD cerca de 8-9/4-5 mmHg menores que sujeitos com aptidão baixa.

\section{CONSIDERAÇÕES FINAIS}

Como vimos o STRONG by Zumba usa o "treinamento intermitente" (que usa a música para direcionar os movimentos e as intensidades), junto com os princípios de treinamento de alta intensidade, para induzir períodos alternados de alta e baixa intensidade, característicos do metabolismo anaeróbico e tem como objetivo manter as intensidades adequadas, usando tanto os sistemas aeróbicos e anaeróbicos, com base no formato de aula que usa os 4 quadrantes, no qual suas intensidades são reguladas pela seleção de movimentos, frequência e colocação de intervalos nos quadrantes e tipos de intervalos (colinas, picos e vales, etc.), podendo ser uma boa ferramenta para avaliar intensidade por meio da Frequência Cardíaca (FC) e suas alterações no sistema cardiovascular.

Para atender os objetivos propostos do estudo, foi verificado as respostas da Frequência Cardíaca (FC) e da Pressão Arterial (PA) em mulheres praticantes de Strong By Zumba ${ }^{\text {Twe }}$ em uma sessão do exercício. Constatouse que as participantes apresentaram diferença significativa nos valores da FC durante e após o exercício quando comparado a primeira avaliação antes do exercício físico proposto, além disso, apesar deste estudo não ter acompanhado a médio prazo a cinética dos níveis pressóricos durante várias sessões de treinos da modalidade, e de não ter apresentado diferença significativa, houve uma diminuição da Pressão Arterial Média (PAM) pós exercício, indicando possível regulação/manutenção da PA induzida pelo treino.

Desta forma, com base nos resultados encontrados neste estudo, nota-se que a modalidade de Strong By Zumba $^{\text {rx }}$ pode ser uma boa aliada na melhora da resposta cardiovascular e do condicionamento físico de seus praticantes. Além disto, esta modalidade, assim como as atividades físicas em grupo em geral, promove a integração social e terapia motivacional, se tornando uma atividade física favorável para a promoção da saúde.

No entanto, faz-se necessário novos estudos para elucidar os seus efeitos desta atividade em diferentes tipos de populações, a fim de preencher as lacunas existentes. Para os próximos estudos, além dos parâmetros avaliados aqui, seria interessante analisar os valores da hipotensão pós-exercício, da FC de repouso como indicadora de aptidão física e calcular o duplo-produto cardíaco durante várias sessões de exercícios em um prazo prolongado para melhor avaliação da resposta cardiorrespiratório, bem como comparar grupos sedentários ou não-praticantes com praticantes da modalidade. É perceptível a escassez de artigos relacionados ao tema proposto, sendo assim, faz-se necessário mais estudos a fim de caracterizar e analisar os efeitos a longo prazo desta modalidade.

\section{REFERÊNCIAS}

American College of Sports Medicine. (2011). Quantity and quality of exercise for developing and maintaining cardiorespiratory musculoskeletal, and neuromotor fitness in apparently healthy adults: guidance for prescribing exercise. Medicine \& science in sport in exercise, 43(7): 1334-1359. 
Araneta, M. R., Tanori, D. (2015). Benefits of zumba fitness among sedentary adults with components of 454 the metabolic syndrome: a pilot study. J Sports Med Phys Fitness. 55(10): 1227-33.

Araujo, J. T. M., Rocha, C. F., Farias, G. M. C., Cruz, R. S., Assunção, J. J. C., Silva, H. J. A., Souza, M. C. (2017). Experiência de mulheres com fibromialgia que praticam zumba. Relato de casos. Rev. dor, São Paulo. 18(3), 266-26. http://dx.doi.org/10.5935/1806-0013.20170113

Barretoi, P. S. (2011). Aderência a longo prazo ao exercício: a relação com a aptidão funcional e a motivação pessoal entre mulheres idosas independentes residentes na comunidade. Rev. Bras. Ciênc. Esporte, Porto Alegre. 33(1), 193-206.

Cugusi, L., Wilson, B., Serpe, R., Medda, A., Deidda, M., Gabba, S., Mercuro, G. (2016). Cardiovascular effects, body composition, quality of life and pain after a zumba fitness program in italian overweight women.J Sports Med Phys Fitness. 3(1): 328-35.

Dada, R. F., Branco, B. H. M., Terra, C. M. O., Lazarin, S. P. B., Hintze, L. J., Junior, N. N. (2018). Estado nutricional e risco cardiometabólico em mulheres: relação com componentes usuais e não usuais da composição corporal. $J$. Phys. Educ.; 29(1):2935. http://dx.doi.org/10.4025/jphyseduc.v29i1.2935

Garber, C.E., Blissmer, B., Deschenes, M.R., Franklin, B.A., Lamonte, M.J., Lee, I.M., Nieman, D.C., Swain, D.P. (2011). Quantity and quality of exercise for developing and maintaining cardiorespiratory musculoskeletal, and neuromotor fitness in apparently healthy adults: guidance for prescribing exercise. Med Sci Sports Exerc. 43(7):1334-59. doi: http://dx.doi.org10.1249/MSS.0b013e318213fefb.

Malaquias, M.V.B., Gomes, M.A.M., Nobre, F., Alessi, A., Feitosa, A.D e Coelho, E.B. (2016). $7^{\text {a }}$ Diretriz Brasileira de Hipertensão Arterial: Capítulo 2 - Diagnóstico e Classificação. Arquivos Brasileiros de Cardiologia, 107 (3, Suppl. 3), 7-13. https://doi.org/10.5935/abc.20160152

Heil, D. P. (2001). Diretrizes do American College of Sports Medicine para teste e prescrição de exercícios. Rev.Med. e Ciên. Esportes e Exercícios. 33(2): 343.

Kokkinos, P., Narayan, P. (2019). Cardiorespiratory Fitness in Cardiometabolic Diseases: Prevention and Management in Clinical Practice. Springer Cham.

Kraemer, W.J., Fleck, S.J., Deschenes, M.R. (2015). Exercise Physiology: Integrating Theory and Application. 2ed. Lippincott Williams \& Wilkins; Philadelphia.

Ljubojević, A. L., Jakovljević, V., Popržen, M. (2014). Effects of Zumba Fitness program on body composition of women. SportLogia, 10(1), 29-33. doi: http://dx.doi.org/10.5550/sgia.141001.en.004L

Oliveira, D.V., Favero, P.F., Codonhato, R., Moreira, C.R., Antunes, M.D., \& Nascimento Júnior, J.R.A (2017). Investigação dos fatores psicológicos e emocionais de idosos frequentadores de clubes de dança de salão. Rev. bras. geriatr. gerontol. Rio de Janeiro. 20(6):. 797-804 http://dx.doi.org/10.1590/1981-22562017020.170089

Pontes-Júnior, F.L., Prestes, J., Leite, R.D., Rodriguez, D. (2011). Influência do treinamento aeróbio nos mecanismos fisiopatológicos da hipertensão arterial sistêmica. Rev. Bras. Ciênc. Esporte. 32(2-4): 229-244. http://dx.doi.org /10.1590/S0101-32892010000200016.

Roy B., \& Mandal S. (2017). Effect of strong by Zumba on waist hip ratio of middle-aged sedentary females. International Journal of Physical Education, Sports and Health. 4(3): 379-381.

Strong By Zumba (2017). Manual de treinamento para instrutor. São Paulo, Brasil. Disponível em https://strong.z umba.com/pt-BR

Triani, F.S., Lima, V.P., Neto, V.G.C., Monteiro, E.R. (2018). Correlação Entre Índice de Massa Corporal, Potência Muscular e Consumo de Oxigênio de Estudantes de Educação Física. J Health Sci., 20(1), 29-33. doi: http://d x.doi.org/10.17921/2447-8938.2018v20n1p29-33

Triviños A.N.S. (1987). Introdução à pesquisa em ciências sociais: a pesquisa qualitativa em educação. São Paulo: Atlas.

Valente, C.C.S. (2018). Comparação da resposta fisiológica, biomecânica e neuromuscular entre as vertentes do fitness: Zumba e Strong by Zumba $a^{\mathrm{Tn}}$. (Dissertação Mestrado em Ciências do Desporto), Guarda: Escola Superior de Educação, Comunicação e Desporto, do Instituto Politécnico da Guarda.

Vendramin, B., Bergamin, M., Gobbo, S., Cugusi, L., Duregon, F., Bullo, V., Ermolao, A. (2016). Health Benefits of Zumba fitness training: a systematic review, $P M \mho R, 8(12) 1181-1200$,. doi: http://dx.doi.org/10.1016/j.pmr j.2016.06.010.

Vlatch, S. (2017). Strong Group Ex: Kick Up the Intensity. (high-intensity interval training, Strong by Zumba) (Group Exercise Moves/Anatomy of a Class). American Fitness, 35(4); 52-4. 
Yatsuya, H., Li, Y., Hilawe, E.H., Ota, A., Wang, C., Chiang, C.,... Aoyama A (2014). Global trend in overweight and obesity and its association with cardiovascular disease incidence. Circ J. 78(12): 2807-2818. https://doi.org/1 0.1253/circj.CJ-14-0850

\section{Notas}

1 A Zumba é a maior marca de dança registrada em todo o mundo, que cativa os participantes devido aos ritmos latinos com movimentos e coreografias simples e fáceis de acompanhar. As aulas de zumba são atreladas aos princípios básicos do treino aeróbico, força, resistência muscular e treino intervalado, aumentando, consequentemente, o gasto calórico, a tonificação muscular e a aptidão cardiorrespiratória, sendo considerada uma atividade que promove melhorias na aptidão física relacionada à saúde. 